Elsevier Editorial System(tm) for Nuclear Inst. and Methods in Physics Research, A Manuscript Draft

Manuscript Number:

Title: Bi-spectral extraction through elliptic neutron guides

Article Type: Research Paper

Section/Category: Accelerators, Beam Handling and Targets

Keywords: bi-spectral neutron beam extraction; neutron instrumentation; supermirror; European Spallation Source; elliptic neutron guide; ray-tracing simulations; ESS; McStas; VITESS; iFit

Corresponding Author: Mr. Henrik Jacobsen, B.sc.

Corresponding Author's Institution: Niels Bohr Institute

First Author: Henrik Jacobsen, B.sc.

Order of Authors: Henrik Jacobsen, B.sc.; Klaus Lieutenant; Carolin Zendler; Kim Lefmann

Abstract: In this article we present the results of investigating a suggested guide extraction system utilizing both a thermal and a cold moderator at the same time, the so-called bi-spectral extraction. Here, the thermal moderator has line of sight to the sample position, and the neutrons from the cold source are reflected by a supermirror towards the sample.

The work is motivated by the construction of the European Spallation Source (ESS) but the results are general and can be used at any neutron source. Due to the long pulse structure, most instruments at ESS will be long, often exceeding $50 \mathrm{~m}$ from moderator to detector. We therefore investigate the performance of bi-spectral extraction for instrument lengths of $30 \mathrm{~m}, 56 \mathrm{~m}, 81 \mathrm{~m}$ and $156 \mathrm{~m}$. In all these cases, our results show that we can utilize both moderators (and thus high intensity in a wide wavelength band) in the same instrument at a cost of flux of 5-25\% for neutrons with wavelength larger than $1 \AA$ A. In general, the divergence distribution is smooth at the sample position. 


\title{
Bi-spectral extraction through elliptic neutron guides
}

\author{
Henrik Jacobsen ${ }^{\mathrm{a}, \mathrm{b}, *}$, Klaus Lieutenant ${ }^{\mathrm{c}}$, Carolin Zendler $^{\mathrm{c}}$, Kim Lefmann $^{\mathrm{a}, \mathrm{b}}$ \\ ${ }^{a}$ Nanoscience center and eScience center, Niels Bohr Institute, University of Copenhagen \\ ${ }^{b}$ ESS design update program, Denmark \\ ${ }^{c}$ Helmholtz-Zentrum Berlin, Hahn-Meitner-Platz 1, D-14109 Berlin, Germany
}

\begin{abstract}
In this article we present the results of investigating a suggested guide extraction system utilizing both a thermal and a cold moderator at the same time, the so-called bi-spectral extraction. Here, the thermal moderator has line of sight to the sample position, and the neutrons from the cold source are reflected by a supermirror towards the sample.

The work is motivated by the construction of the European Spallation Source (ESS) but the results are general and can be used at any neutron source. Due to the long pulse structure, most instruments at ESS will be long, often exceeding $50 \mathrm{~m}$ from moderator to detector. We therefore investigate the performance of bi-spectral extraction for instrument lengths of $30 \mathrm{~m}, 56$ $\mathrm{m}, 81 \mathrm{~m}$ and $156 \mathrm{~m}$. In all these cases, our results show that we can utilize both moderators (and thus high intensity in a wide wavelength band) in the same instrument at a cost of flux of 5-25\% for neutrons with wavelength larger than $1 \AA$. In general, the divergence distribution is smooth at the sample position for all wavelengths.
\end{abstract}

Keywords:

\footnotetext{
*Corresponding author

Email address: hjacobse@fys.ku.dk (Henrik Jacobsen) 
bi-spectral neutron beam extraction, neutron instrumentation, supermirror, European Spallation Source, elliptic neutron guide, ray-tracing simulations, ESS, McStas, VITESS, iFit

\section{Introduction}

The long-pulsed European Spallation neutron source, the ESS, is presently in planning [1]. Due to the long pulse, most instruments will be long, exceeding $50 \mathrm{~m}$ from moderator to detector. For the design of instruments for the ESS, much assumed knowledge is presently being re-investigated. One important part is the neutron guide system, where elliptical guides are being considered for many instruments. Much effort is currently put into understanding how elliptic guides transport neutrons [2 44]. A particular challenge is the design of the first few meters; the so called guide extraction system.

Bi-spectral extraction, as first proposed by Mezei and Russina [5-7] is being considered for several instruments. This system is already implemented at the EXED [8] beam line at HZB, and is shown to work well with straight guides. However, there has been much discussion whether bi-spectral extraction will work equally well with elliptic guides, which is the subject of this work.

A sketch of uni- and bi-spectral extraction is given in Fig. 1. For detailed information about uni-spectral extraction, see e.g. the work of Klenø et. al [2].

In the bi-spectral extraction system, a cold and a thermal moderator are located next to each other. A supermirror reflects the neutrons from the cold source into the guide while neutrons from the thermal source are able 

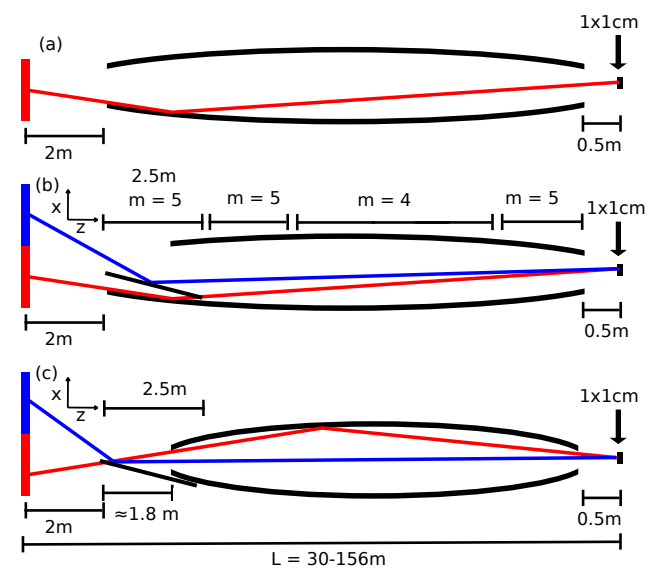

Figure 1: (Color online) Sketch of (a) uni-spectral and (b-c) bi-spectral extraction with elliptic guides. Top view. The minor axes of the ellipses is greatly exaggerated for clarity. (b) illustrates the expected optimal settings with the mirror inside the guide, while (c) illustrates a typical optimum found in this work.

to pass through the mirror into the guide. As discussed below, supermirrors reflect neutrons below a critical scattering vector given by $m q_{c}$, where in our case $m=4$ and $q_{c}=0.0217 \AA^{-1}$ is the critical scattering vector of nickel. Therefore, most of the thermal neutrons pass through the mirror due to their large $k$-vectors and the low absorption in the mirror. Thus the mirror can be seen as a switch between the two moderators, that activates the cold moderator and deactivates the thermal moderator when the wavelength is increased above a certain cross-over wavelength. Ideally, the cross-over should be near $\lambda_{c} \approx 2.5 \AA$ where the brilliance curves of the two moderators meet. This makes it possible to utilize a wide range of incoming neutron wavelengths.

Of course, the cross-over wavelength depends on incident angle of the neutron velocity with respect to the mirror, and is therefore different for 
different parts of the two moderators. So the switching from the thermal to the cold moderator happens gradually near $\lambda=2.5 \AA$, and close to this wavelength, neutrons from both moderators will reach the sample. Even so, it is here worth noting that, for any given wavelength and divergence, the theoretically highest intensity at the sample is the maximum of the intensity of the cold and the thermal source, and not the sum of the two, as one might expect. This follows directly from Liouville's theorem [9]. See also the discussion of (Eq. 4).

\section{Introduction to the simulations}

To investigate the extraction system in detail, we have simulated the setup using the Monte Carlo neutron ray-tracing packages McStas [10, 11] and VITESS [12, 13].

The optimizations and data plotting for McStas data were performed using iFit [14, 15]. The computations were carried out on the 500 core cluster of the ESS Data Management and Software Center [16]. As an example, the optimization and subsequent simulation of the results shown in Fig. 3 took approximately 1 day on a 12 core node of the cluster. For VITESS simulations, the HZB cluster was used [17].

In the following we will briefly outline the instrument, and discuss which parameters are fixed and which are optimized in these simulations.

The moderator characteristics used are the standard ESS sources provided in McStas 1.12c, with a slight modification of the cold source in order to direct the beam to the guide entrance to save simulation time. The temperature of the thermal source is $325 \mathrm{~K}$, that of the cold source is $50 \mathrm{~K}$. The size of 
simulations. The standard description of the reflectivity in version $1.12 \mathrm{c}$ McStas assumes constant reflectivity for $q<q_{c}$ (we here use $R_{0}=0.99$ ), a linear decrease of reflectivity with a certain slope, $\alpha$ (typically $\alpha=3.5$ ) followed by a sharp cutoff with width $W$ around $m q_{c}$, where $1<m<7$ is the $m$-value of the mirror:

$$
R(q)=R_{0}\left(1-\alpha\left(q-q_{c}\right)\right) \tanh \left(\frac{q-m q_{c}}{W}\right) .
$$

It turns out that the linear decrease in this model is too simple and does not accurately describe real mirrors. To improve the description, reflectivity curves for 7 state-of-the-art mirrors with different $m$-values, provided by Swiss Neutronics [18], were fitted to the following generalization of the model

$$
\begin{aligned}
& R(q)= \\
& R_{0}\left(1-\alpha\left(q-q_{c}\right)+\beta\left(q-q_{c}\right)^{2}\right) \tanh \left(\frac{q-m q_{c}}{W}\right) .
\end{aligned}
$$

${ }_{67}$ The values of $\alpha, \beta$ and $W$ were extracted and found to a good approximation 68 
that accurately describes real mirrors and requires only $m$ as input. The reflectivity curves as function of $q$ are shown along with the data for $m=2$, $m=3, m=4, m=5, m=6$ and $m=7$ in Fig. 2. It is here worth noting that, contrary to the version 1.12c McStas model, larger values of $m$ do not always lead to more neutrons being reflected by the mirror: although the reflectivity is non-zero for larger values of $q$, it is significantly lower for low values of $q$.

This model will be the default in McStas 2.1 [11], and can be used in the VITESS guide modules by generating reflectivity files with the according tool in VITESS 3 or higher [13]. In the sm_ensemble module, the reflectivity and attenuation have been changed to match the McStas models, which will be available in VITESS from version 3.1 onwards.

Based on these considerations, an optimization of the optimal coating for a $156 \mathrm{~m}$ instrument showed that optimal transfer of neutrons is achieved when the coating of the guide is $m=5$ for the first and last 15 parts of the guide near the ends and $m=4$ in the middle. For a $156 \mathrm{~m}$ instrument, the $m=5$ coating covers approximately the first $15 \mathrm{~m}$ and last $12 \mathrm{~m}$. For a $56 \mathrm{~m}$ instrument, the $m=5$ coating covers approximately the first $7 \mathrm{~m}$ and last $5 \mathrm{~m}$. Of course it is very expensive to construct a $156 \mathrm{~m}$ guide with $m \geq 4$ throughout. For most of the guide, lower $m$-values can be used with essentially no loss of neutrons, as shown by Refs. [19, 20]. Optimizing the cost of the guide is, however, not the purpose of this paper, and we therefore use these high $m$-values.

The optimal values for the horizontal and vertical focal points and small axis widths all depend on the exact set-up and figure of merit for the sim- 
ulations, and therefore need to be optimized. In some of the optimizations, the optimal settings for the neutrons from the cold moderator will make the guide opening width/height very small, drastically reducing the intensity of the neutrons from the thermal source. To compensate for this, the start position of the guide has also been optimized. This effect is illustrated in Fig. 1 c.

Perhaps the most important component in these simulations is the mirror. The McStas Mirror component does not take absorption into account, and therefore a new mirror component has been written (curved_mirror). Initially, the coating was chosen to be $m=5$, but with the description of the coating according to (Eq. 2), $m=4$ performs better than $m=5$, and has therefore been used. The mirror is modeled as a $t_{c}=10 \mu \mathrm{m}$ thin supermirror layer with $50 \%$ Titanium and $50 \%$ Nickel on top of a $t_{s}=0.5 \mathrm{~mm}$ thick sapphire substrate, in which refraction and attenuation due to absorption and inelastic incoherent scattering are taken into account [21]. For details see Fig. 2. As an example, a $1 \AA$ neutron reaching the mirror with an angle $\theta_{1}=1.25^{\circ}$ will be attenuated by approximately $7 \%$.

It should here be noted that it is possible that some of the mirror is located inside the guide, where it fills out the guide completely in the vertical direction (out of the plane in Fig. 1 (b) ). This is not supported by the standard guide components in McStas. To implement this, an elliptic guide wall component (elliptic_mirror) has been written and is used to model each wall of the guide for the first few meters. As illustrated in Fig. 1, the guide wall facing the cold source is shorter than the others, to allow the neutrons from the cold source to reach the mirror. This means that the order 
of the components is not uniquely defined, as is normally the case in McStas. Correct propagation of the neutrons is thus realized by a generalization of the method described in [22].

We have investigated the effect of curving the mirror, and have come to the conclusion that almost no gains are possible. We have also tried varying the $m$-value along the mirror, also with no gains. To limit the investigated parameter space, we therefore work with a flat mirror with the same $m$-value throughout.

For the VITESS simulation, the same moderator and material characteristics were used. The only difference is that the thickness of the supermirror layer on the mirror is not explicitly considered. The module supermirror_ensemble has been used to simulate the mirror and the guide system around the mirror.

We have analyzed this set-up using several different figures of merit. First of all, the instrument will be compared to the standard uni-spectral extraction. In general, the usable wavelength bands, $\delta \lambda$, depend on the length of the instrument and the time structure of the source according to

$$
\delta \lambda=\frac{T}{\alpha L},
$$

where $T$ is the moderator period ( $T=71.4 \mathrm{~ms}$ for ESS), $L$ is the length of the instrument and $\alpha=m_{\mathrm{n}} / h=252.7 \mu \mathrm{s} / \mathrm{m} / \AA$. We here investigate 4 of the standard lengths considered for ESS [23] : $30 \mathrm{~m}, 56 \mathrm{~m}, 81 \mathrm{~m}$ and 156 $\mathrm{m}$, corresponding to $\delta \lambda=9.4,5.0,3.5$ and $1.8 \AA$, respectively. The main question is how well the set-up performs for cold neutrons. We have chosen to restrict the wavelength bands somewhat for the short instruments, and have thus optimized the following 'cold' wavelength bands: $30 \mathrm{~m}$ : $3.0-7.5$ 

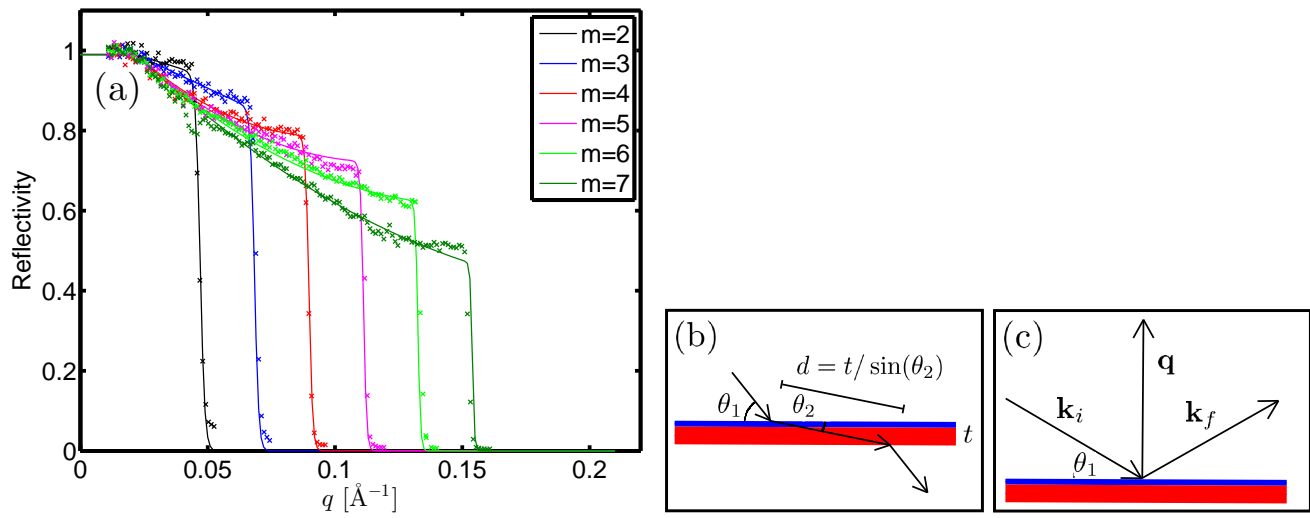

Figure 2: (Color online) Model of the mirror. (a) the reflectivity for different $m$-values as function of scattering vector, $q$. The data points are measurements by Swiss Neutronics and the solid lines are the model (Eq. 21) (b) and (c): The mirror consists of a thin coating (blue) on top of a thicker substrate (red). (b) illustrates how absorption and refraction are taken into account, while (c) illustrates reflections.

$\AA, 56 \mathrm{~m}: 3.0-7.5 \AA, 81 \mathrm{~m}: 3.25-6.75 \AA, 156 \mathrm{~m}: 4.1-5.9 \AA$.

The virtue of bi-spectral extraction is the possibility to utilize a wide wavelength band. We have therefore also optimized the instrument in the 'full' wavelength band $0.75-7.25 \AA$. Finally, the overlap region near $2.5 \AA$, where the brilliance is the same for the two moderators, is of special interest. We have therefore also optimized the set-up within 1-4 $\AA$, here named the 'bi-spectral' wavelength band.

Each of these optimizations have been carried out for three different limits for the divergence at the sample position, as previously studied by e.g. Ref. [2] : $\pm 0.5^{\circ}, \pm 1.0^{\circ}$ and $\pm 2.0^{\circ}$. This gives a total of 36 optimizations of bispectral extraction and 24 optimizations of uni-spectral extraction.

Optimizing absolute intensities does not produce satisfactory results, as the intensity of $1.5 \AA$ is much higher than e.g. $5 \AA$ neutrons. We have 
therefore optimized the brilliance transfer, $B(\lambda, D)$ instead. Brilliance is defined as number of neutrons per second, per square centimeter, within a wavelength band $\lambda$, within a divergence limit $(D)$. Brilliance transfer, then, is the ratio of brilliance at the sample and the source. The virtue of this is that all wavelengths are weighted equally.

For any given $\lambda$ and $D$, the bi-spectral source brilliance $B_{\mathrm{bi}}(\lambda, D)$ is the maximal brilliance of the two moderators. Naming the brilliance of the cold source $B_{\mathrm{c}}(\lambda, D)$ and that of the thermal source $B_{\mathrm{t}}(\lambda, D)$, we thus have

$$
B_{\mathrm{bi}}(\lambda, D)=\left\{\begin{array}{lll}
B_{\mathrm{t}}(\lambda, D) & \text { for } & \lambda<\lambda_{c} \\
B_{\mathrm{c}}(\lambda, D) & \text { for } & \lambda>\lambda_{c} .
\end{array}\right.
$$

We note again that Liouville's theorem [9] states that the brilliance transfer can never exceed $100 \%$. This makes $B(\lambda, D)$ a direct measure of the quality of the guide system.

The majority of the results will be given in terms of brilliance transfer instead of absolute intensities, and are therefore of general validity, also for other sources than ESS.

\section{Results}

\subsection{Wavelength distribution}

Fig. 3 shows an example of the simulated intensity as a function of wavelength on a $1 \times 1 \mathrm{~cm}^{2}$ sample for neutrons with divergence less than $0.5^{\circ}$. The best results that can be obtained with uni-spectral extraction are shown for comparison. 


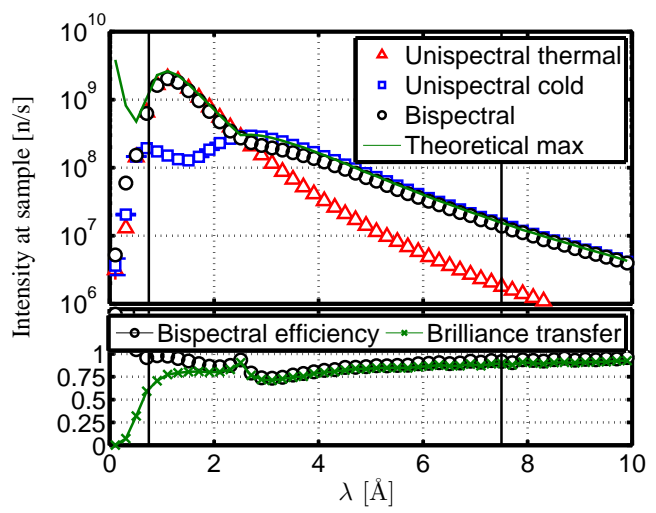

Figure 3: (Color online) The wavelength distribution at the sample, comparing the performance of bi-spectral extraction to a normal uni-spectral extraction system for a $156 \mathrm{~m}$ instrument for neutrons with divergence within $\pm 0.5^{\circ}$. The vertical lines show the limits of the wavelength band used in the optimizations. The lower panel shows brilliance transfer and efficiency compared to uni-spectral extraction.

In the lower panel, the brilliance transfer and efficiency are plotted. Efficiency at a given wavelength is defined as the performance compared to an optimal uni-spectral elliptic guide, and mainly serves to judge the performance below $1 \AA$, where the brilliance transfer drops quickly to zero.

In this example we see that it is possible to obtain brilliance transfers exceeding $75 \%$ for neutrons with wavelengths larger than $0.75 \AA$. For neutrons with wavelengths larger than $6 \AA$, the brilliance transfer reaches more than $90 \%$. These results depend slightly on which wavelength band has been optimized. Before investigating other wavelength bands and instrument lengths, we validate the simulations by comparing McStas and VITESS simulations, as shown in Fig. 4. In this comparison only, the absorption in the coating of the mirror is neglected in the McStas simulation. It is seen that the agreement between McStas and VITESS is within $3 \%$ except at $\lambda<0.6 \AA$. 


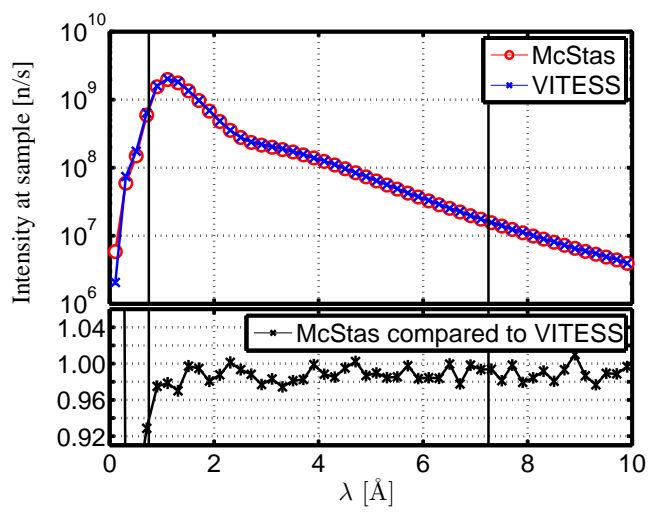

Figure 4: (Color online) The wavelength distribution at the sample for a $156 \mathrm{~m}$ instrument for neutrons with divergence within $\pm 0.5^{\circ}$, comparing McStas and VITESS simulations. The vertical lines show the limits of the wavelength band used in the optimizations. The lower panel shows the ratio of intensity of McStas to VITESS simulations.

In Fig. [5 we give an overview of the performance for all the optimizations mentioned above, showing brilliance transfer as function of wavelength for the 4 different instrument lengths and 3 divergence limits. Each figure contains five graphs: (-) and (- -) show the performance of the thermal and cold unispectral, respectively, when compared to the Liouville limit for the bi-spectral extraction. The three other graphs show the results when optimizing for the 'cold' (o), 'full' $(\triangle)$ and 'bi-spectral' $(\square)$ wavelength bands, respectively. Much information can be extracted from Fig. 5. A general feature is that for low divergent neutrons, it is possible to obtain brilliance transfers exceeding $70 \%$ for neutrons with wavelength $1 \AA$ or higher. The brilliance transfer of thermal neutrons can be increased at the cost of cold neutrons and vice versa. It is not quite possible to reach the performance of two combined uni-spectral sources throughout the interesting wavelength band, but we can reach $75 \%$ in the overlap region and up to $95 \%$ elsewhere. 
For neutrons with divergence larger than $\pm 0.5^{\circ}$, brilliance transfers within $50-75 \%$ can be obtained.

In some cases, the optimal settings found by the optimizer is the same for all three figures of merit, and thus one or two of the data sets are not visible.

\subsection{Divergence distribution}

Let us now look closer at the neutrons getting through the guide. We will focus on the set-ups that give best overall brilliance transfer of low divergent neutrons $\left( \pm 0.5^{\circ}\right)$, i.e. we show the results of the following optimizations: 30 m: 'cold', 56 m: 'cold', $81 \mathrm{~m}$ : 'bi-spectral', $156 \mathrm{~m}$ : 'full'. The divergence of the neutrons should ideally be smooth and symmetric. In Fig. 6 we show the divergence for three different $0.01 \AA$ wide wavelength bands, centered on the following wavelengths: $1.5 \AA(\circ), 2.5 \AA(\square)$ and $5.0 \AA(\times)$. In the plot of $x(y)$ divergence, the neutrons with $y(x)$ divergence larger than than $0.5^{\circ}$ have been removed.

There is some structure in the divergence distribution, especially for the $30 \mathrm{~m}$ instrument, but in general, the divergence is quite smooth within the chosen limits. In some cases, there are a lot of unwanted neutrons, i.e. neutrons with divergence larger than the required limits. These will of course have to be removed, e.g. by replacing the last few meters of the guide with absorbing material, by use of collimators or slits in the guide or by further optimizations. Modification of this detail is, however, not the purpose of this work.

There are differences between the divergence distribution in the horizontal $(x)$ and vertical $(y)$ direction. There are three reasons for this. The main 

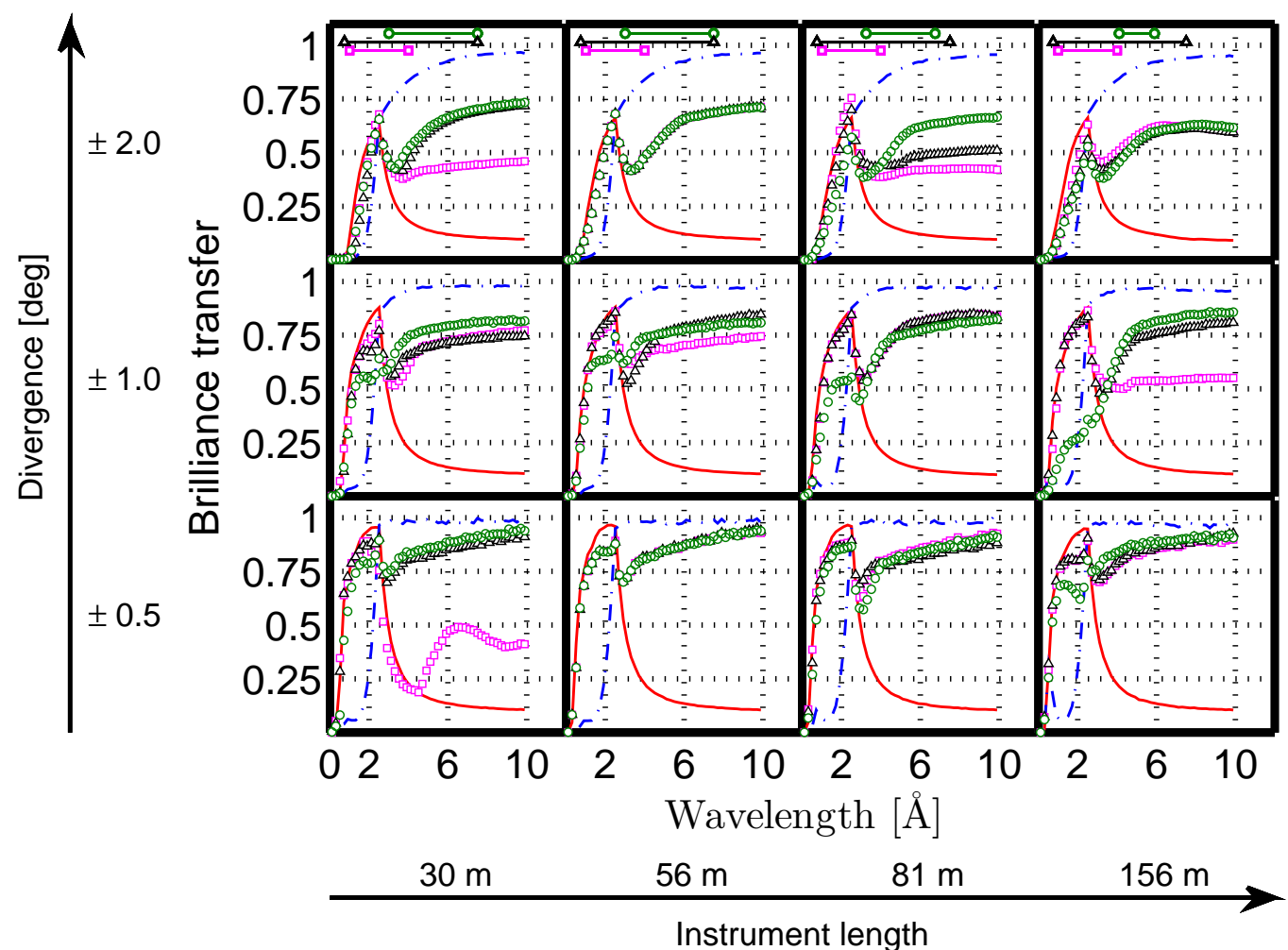

Figure 5: (Color online) Brilliance transfer distribution, optimized for 3 different wavelength bands. (-) and (- -) show the performance of the thermal and cold uni-spectral, respectively, when compared to the Liouville limit for the bi-spectral extraction. The three other graphs show the results when optimizing for the 'cold' (o), 'full' $(\triangle)$ and 'bispectral' $(\square)$ wavelength bands, respectively. The horizontal lines in the top indicate these wavelength bands. 


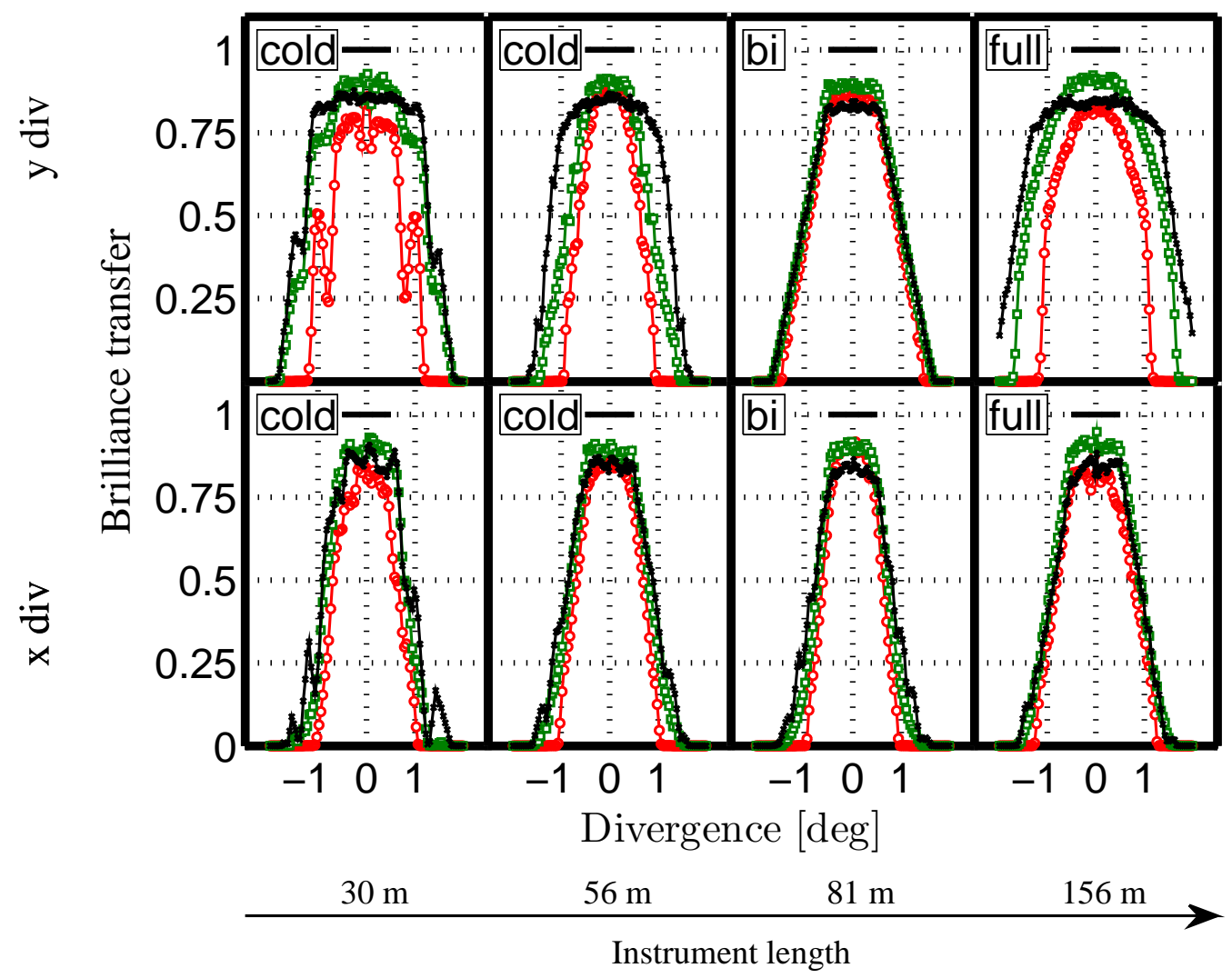

Figure 6: (Color online) Horizontal $(x)$ and vertical $(y)$ divergence for the set-ups with best overall performance, for neutrons with the following wavelengths, $1.5 \AA$ ( $)$ ), $2.5 \AA$ $(\square)$ and $5.0 \AA(\times)$. The horizontal lines show the divergence for which the set-up has been optimized $\left( \pm 0.5^{\circ}\right)$, and the text indicates the wavelength band that has been optimized: $30 \mathrm{~m}$ : 'cold', $56 \mathrm{~m}$ : 'cold', $81 \mathrm{~m}$ : 'bi-spectral', $156 \mathrm{~m}$ : 'full'. The cross section of the guides is rectangular. 
reason is that, contrary to e.g. Ref. [2], the cross-section of the guide is not forced to be square. This extra freedom in parameter space has been added because the horizontal and vertical directions are not a priori equal. Secondly, the mirror distorts the divergence in the horizontal direction, and thirdly gravity has a small effect on the vertical direction.

In Fig. 7, the same results are shown for an optimization in which the cross section of the guide has been forced to be square. Here, the divergence in the horizontal-direction is not at all pretty, and the intensities are smaller by $5-10 \%$. The loss in intensity can be tolerated, but the uneven divergence distribution could be a problem. We can thus conclude that to limit the negative effects of the mirror, the guide cross section must be rectangular instead of square.

\subsection{Acceptance diagrams}

In Fig. 8 we further investigate the $156 \mathrm{~m}$ instrument shown in Fig. 3 and 6, i.e. optimized for low divergence within the 'full' wavelength band. We focus on 3 wavelengths: $1.5 \AA$ (top), $2.5 \AA$ (center) and $5 \AA$ (bottom). Each figure shows 4 plots: (a) 2 d divergence, (b) horizontal acceptance diagram (divergence vs position), (c) vertical acceptance diagram, (d) position. The black boxes indicate the sample position and divergence limit. In the dimensions not shown in each figure, only the neutrons that reach the sample with divergence within $\pm 0.5^{\circ}$ are counted. In the horizontal acceptance diagram, for example, neutrons with vertical divergence larger than $\pm 0.5^{\circ}$ are removed, while in the divergence monitor neutrons outside the $1 \times 1$ $\mathrm{cm}^{2}$ sample position are removed. All the monitors have been normalized to brilliance transfer. 


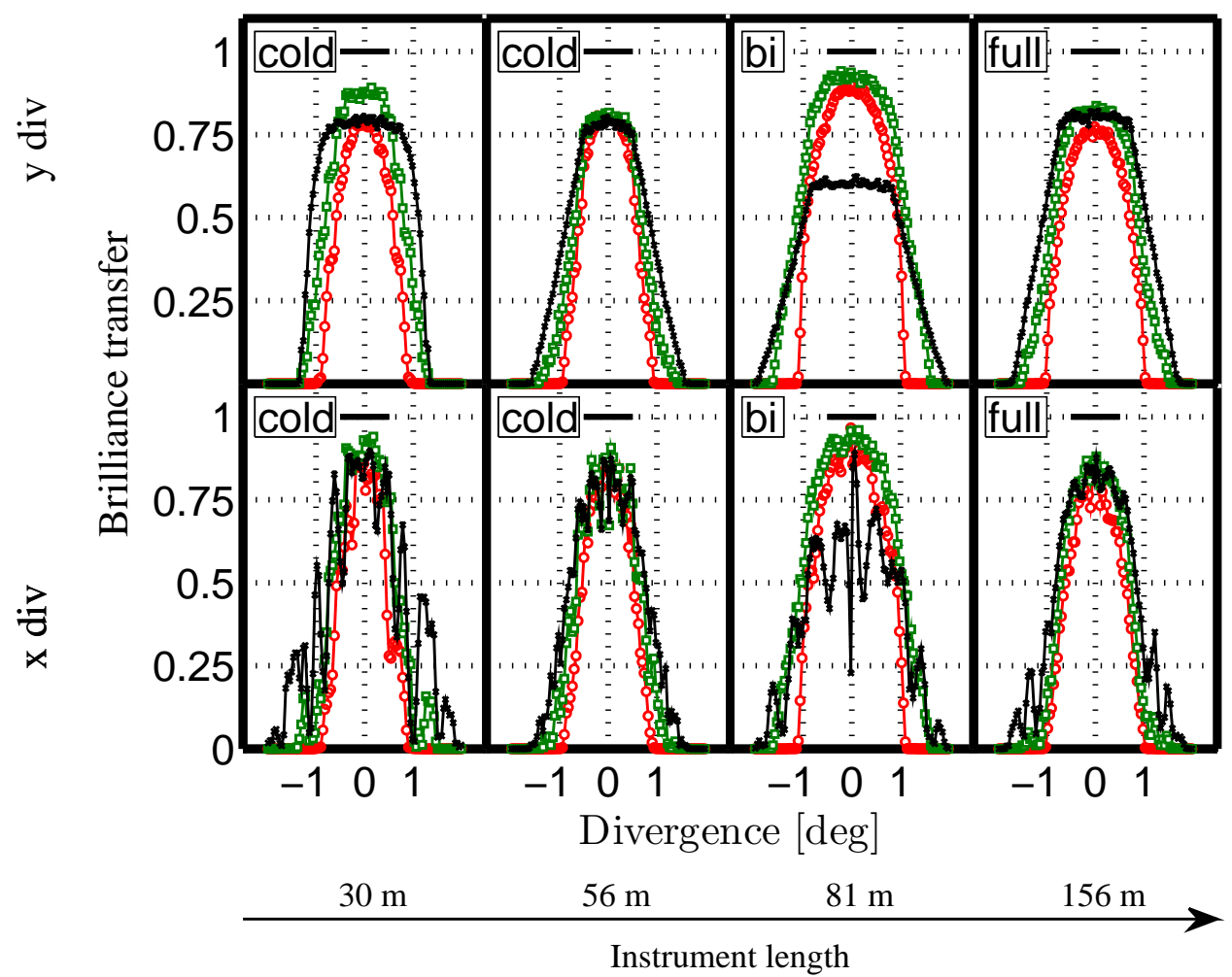

Figure 7: (Color online) Horizontal $(x)$ and vertical $(y)$ divergence for the set-ups with best overall performance when the guide has a square cross section, for neutrons with the following wavelengths, $1.5 \AA(\circ), 2.5 \AA(\square)$ and $5.0 \AA(\times)$. The horizontal lines show the divergence for which the set-up has been optimized $\left( \pm 0.5^{\circ}\right)$, and the text indicates the wavelength band that has been optimized: $30 \mathrm{~m}$ : 'cold', $56 \mathrm{~m}$ : 'cold', $81 \mathrm{~m}$ : 'bi-spectral', 156 m: 'full'. 

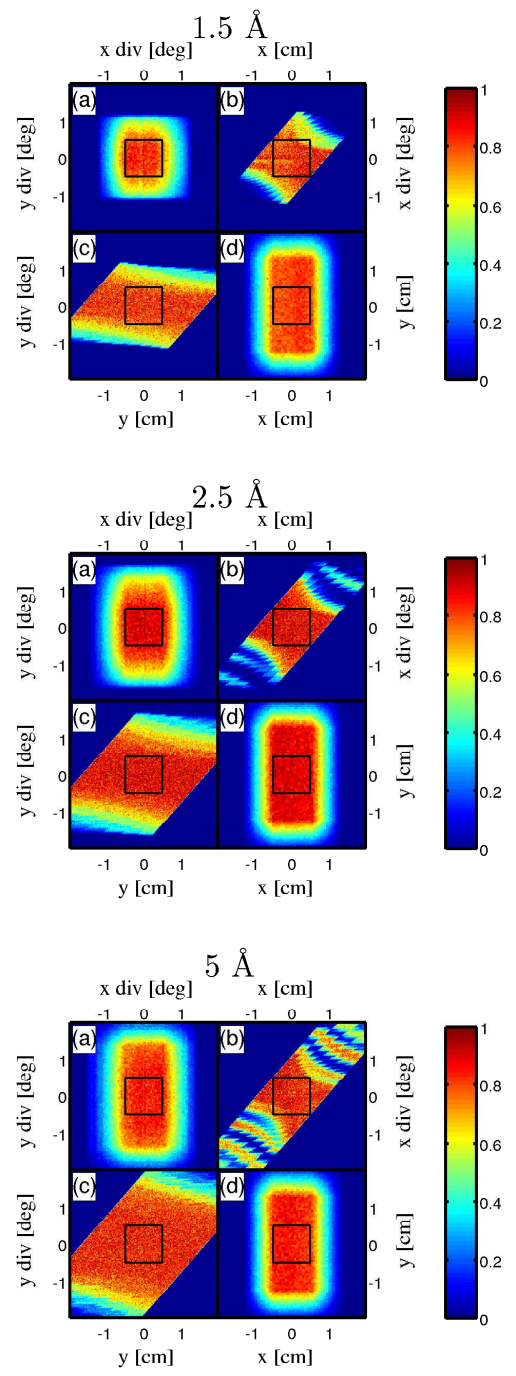

Figure 8: (Color online) Investigation of the properties of neutrons getting through the guide for a $156 \mathrm{~m}$ extraction optimized for low divergent neutrons within the 'full' wavelength band. (a) 2d divergence, (b) horizontal acceptance diagram (divergence vs position), (c) vertical acceptance diagram, (d) position. The black boxes indicate the sample position and divergence limit. In the dimensions not shown in each figure, only the neutrons that hit the sample with horizontal $(x)$ and vertical $(y)$ divergence within $\pm 0.5^{\circ}$ are counted. In the horizontal acceptance diagrams (b), for example, neutrons with vertical divergence larger than $\pm 0.5^{\circ}$ are removed. 
It is seen that the beam profile is smooth at the sample position for all wavelengths. It should be noted that many unwanted neutrons reach the sample position.

The parameters for this set-up (156 m, low divergence, optimized for 'full' wavelength band) are the following, where all positions are given relative to the center of the thermal moderator: start of guide: $3.86 \mathrm{~m}$, first horizontal focus point: $2.80 \mathrm{~m}$, second horizontal focus point: $156.0 \mathrm{~m}$, largest width of the guide: $16.5 \mathrm{~cm}$, first vertical focus point: $-0.05 \mathrm{~m}$, second vertical focus point: $156.6 \mathrm{~m}$, height of guide: $20.1 \mathrm{~cm}$, center position of mirror: $-0.9 \pm 0.5 \mathrm{~cm}$, inclination of mirror: $1.1 \pm 0.2^{\circ}$. The uncertainties in the last two numbers are estimates on what error can be tolerated without significant loss of neutrons. This has been found by simulating the specific set-up with varying values of the two parameters. The effect of misaligning the guide has been studied elsewhere [24].

Thus, the dimensions of the guide at the start are $2.7 \times 6.4 \mathrm{~cm}^{2}$, while at the exit they are $1.9 \times 3.3 \mathrm{~cm}^{2}$. It is interesting to note that the optimal position of the mirror is outside the guide as shown in Fig. 1 (c); this was not anticipated from the first results of this work.

In Fig. 9 we show the same plots for the $30 \mathrm{~m}$ instrument shown in Fig. 3 (i.e. optimized for low divergent neutrons). The neutrons reaching the sample with the wanted divergence in general behave well. A notable exception is the $1.5 \AA$ neutrons, where the intensity is visibly larger on one side of the sample than the other. This is because the path length through the mirror, and therefore the absorption, depends on the incoming angle of the neutrons, which is what determines where the neutrons hit the sample. This 
effect is not seen in longer guides where the neutrons are reflected several times by the guide before reaching the sample [4]. Another effect for 1.5 $\AA$ neutrons is some structure in the divergence distribution. However, this appears quite symmetric and therefore should not be a problem for the $q$ dependent part of the instrument resolution function.

The parameters for this set-up are the following, where all positions are given relative to the center of the thermal moderator: start of guide: 3.56 $\mathrm{m}$, first horizontal focus point: $2.0 \mathrm{~m}$, second horizontal focus point: $30.5 \mathrm{~m}$, largest width of the guide: $5.7 \mathrm{~cm}$, first vertical focus point: $1.7 \mathrm{~m}$, second vertical focus point: $30.7 \mathrm{~m}$, height of guide: $14.5 \mathrm{~cm}$, center position of mirror: $-0.7 \pm 0.5 \mathrm{~cm}$, inclination of mirror: $1.1 \pm 0.2^{\circ}$.

Thus, the dimensions of the guide at the start are $2.6 \times 7.2 \mathrm{~cm}^{2}$, while at the exit they are $2.0 \times 5.8 \mathrm{~cm}^{2}$.

Here, it is worth noting that the width of the guide is quite small.

\section{Discussion and conclusion}

We have investigated bi-spectral extraction through elliptic guides for 4 typical instrument lengths proposed for ESS using McStas and VITESS simulations. Our simulations show that brilliance transfers of more than $75 \%$ can be achieved for neutrons with wavelength larger than 1 A. For cold neutrons, brilliance transfers exceeding $90 \%$ are obtainable.

We have focused on neutrons with relatively low divergence $\left( \pm 0.5^{\circ}\right)$, and have found that the divergence profile at the sample position is smooth, as is required by many instrument designers.

The figures of merit for these simulations are intensity of neutrons at the 

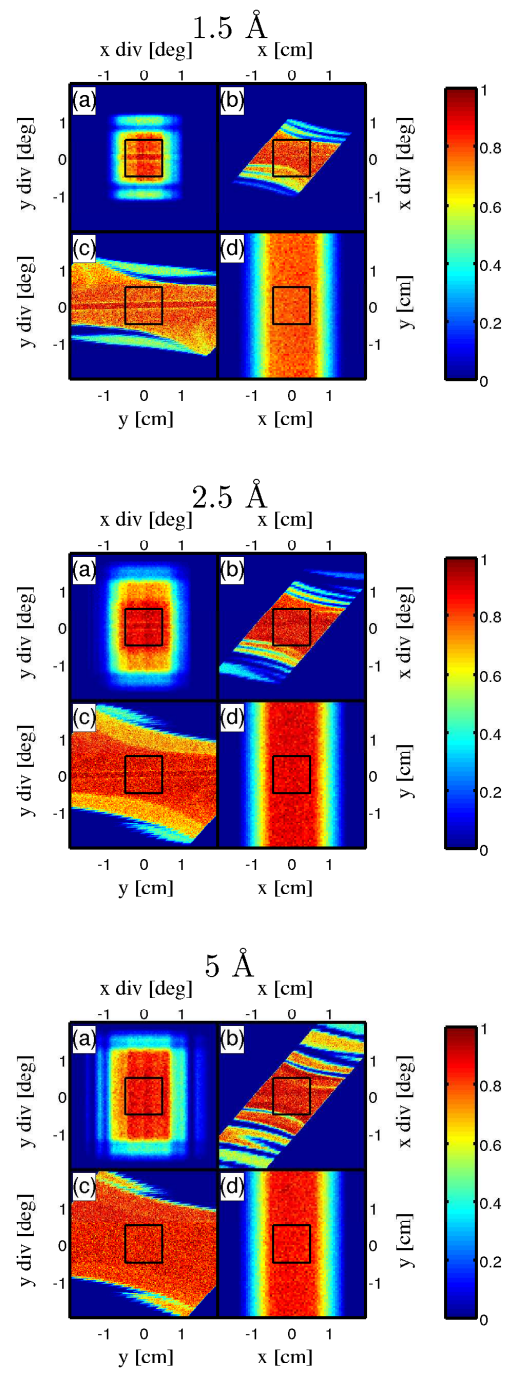

Figure 9: (Color online) Investigation of the properties of neutrons reaching getting through the guide for a $30 \mathrm{~m}$ extraction, optimized for low divergent neutrons within the 'cold' wavelength band. (a) 2d divergence, (b) horizontal acceptance diagram (divergence vs position), (c) vertical acceptance diagram, (d) position. The black boxes indicate the sample position and divergence limit. In the dimensions not shown in each figure, only the neutrons that hit the sample with divergence within $\pm 0.5^{\circ}$ are counted. In the horizontal acceptance diagrams (b), for example, neutrons with vertical divergence larger than $\pm 0.5^{\circ}$ are removed. 
sample position within certain divergence limits. Another important requirement for all instruments is that the background be minimal. Therefore, it is often desired to get out of line of sight. For short instruments this is obviously difficult for both uni- and bi-spectral extraction, but not impossible. For longer instrument, using e.g. a double ellipse and a kink in the guide has been shown to work well with uni-spectral extraction [25]. Our work shows that the beam profile after the guide in general is similar to that of uni-spectral extraction. It should therefore not be a problem to implement a kink e.g. at $30 \mathrm{~m}$ and a second ellipse to get out of line of sight for longer instruments.

Another option that is considered for many instruments is to use a feeder (converging guide and a pinhole) to compress the beam for a chopper at 6 m. Indeed, the recent work presented in Ref. [26] shows that bi-spectral extraction works well with a feeder, with performance nearly reaching that of an elliptic guide. In short, every guide optical trick used by uni-spectral extraction should still work for bi-spectral extraction.

To carry out these simulations, an improved model for reflectivities has been implemented in McStas and VITESS, and two new McStas components have been written and tested: a mirror that correctly takes absorption into account and an elliptic guide wall. We have also, based on Ref. [22], further developed a method to ensure correct propagation of the neutrons when the order of components is not uniquely defined, as is the case here. Finally, we have implemented a general method to include two (or more) different moderators in McStas. The McStas instrument file, these components and files containing the parameters found in the optimizations presented will 
be made available on the McStas website [11] and can also be obtained by contacting the main author.

It is interesting to note that most of the mirror is placed outside the guide: even when the starting parameters for the optimizations were with the mirror firmly inside the guide, as in Fig. 1 b, the optimizer would converge to having the mirror outside the guide, as in Fig. 1 c. Also, our simulations show that the optimal guide set-up is not with a square cross section, but rather a rectangle that is taller than it is wide. If a square cross-section is forced, this decreases the performance significantly.

We can finally conclude that obtaining a wide wavelength band using bispectral extraction is indeed feasible using elliptic guides for both long and short instruments. The beam profile is homogeneous at the sample, and the divergence is smooth and symmetric.

\section{Acknowledgement}

We thank Ken H. Andersen, Pascale Deen, and Kaspar H. Klenø for engaging in discussion on the article subject and commenting on results. We thank Peter K. Willendrup and Emmanuel Farhi for technical help with software and the ESS-DMSC for providing computing power. We thank Swiss Neutronics for providing data for the model of the mirror. We thank the Danish Agency for Research and Innovation and Bundesministerium für Bildung und Forschung (BMBF) for their support through the contribution to the ESS update phase. 


\section{References}

\section{References}

[1] ESS website. http://ess-scandinavia.eu/.

[2] Kaspar Hewitt Klenø, Klaus Lieutenant, Ken H. Andersen, and Kim Lefmann. Systematic performance study of common neutron guide geometries. Nuclear Instruments and Methods in Physics Research Section A: Accelerators, Spectrometers, Detectors and Associated Equipment, 696(0):75-84, 2012.

[3] Phillip M. Bentley, Shane J. Kennedy, Ken H. Andersen, and F. R. David. Correction of Optical Aberrations in Elliptic Neutron Guides. 2001.

[4] L.D. Cussen, D. Nekrassov, C. Zendler, and K. Lieutenant. Multiple reflections in elliptic neutron guide tubes. Nuclear Instruments and Methods in Physics Research Section A: Accelerators, Spectrometers, Detectors and Associated Equipment, pages 1-11, December 2012.

[5] F. Mezei and M. Russina. Neutron-optical component array for the specific spectral shaping of neutron beams or pulses. Patent US, 7030397 B2 (2006).

[6] F. Mezei and M. Russina. Neutronenoptische Bauelementenanordnung zur gezielten 242 spektralen Gestaltung von Neutronenstrahlen oder Pulsen. Patent Germany Amtl. Aktz., 10203591 (2002), 2002. 
[7] F. Mezei. Advances by innovation and building on experience. The ESS project Vol II, New Science and Technology for the 21st Century (2002), 2002 .

[8] K. Lieutenant J. Peters and F Mezei. Monte Carlo simulation of the new time-of-flight powder diffractometer EXED at the Hahn-MeitnerInstitut. Journal of Neutron Research, 14(2):147-165, 2006.

[9] L. D. Landau and E. M. Lifshitz. Statistical Physics, volume 5 of Course of Theoretical Physics. Butterworth-Heinemann, third edition, 1980.

[10] Kim Lefmann and K. Nielsen. McStas, a general software package for neutron ray-tracing simulations. Neutron News, 10/3:20-23, 1999.

[11] McStas website. http://www.mcstas.org/.

[12] Klaus Lieutenant, Geza Zsigmond, Sergey Manoshin, Michael Fromme, Heloisa N Bordallo, Dickon Champion, Judith Peters, and Ferenc Mezei. Neutron instrument simulation and optimization using the software package VITESS. pages 134-145, 2004.

[13] VITESS website. http://www.helmholtz-berlin.de/vitess.

[14] E. Farhi. The iFit data analysis library.

[15] E. Farhi, Y. Debab, and P. Willendrup. iFit. J. Neut. Res., (17), 2012.

[16] DMSC website. http://www.ess-dmsc.eu.

[17] HZB cluster dirac. http://www.helmholtz-berlin.de/angebote/it/dienste/dirac/index

[18] Swiss Neutronics website.http://www. swissneutronics.ch/products/coatings.html. 
[19] Andreas Houben, Werner Schweika, Thomas Brückel, and Richard Dronskowski. New neutron-guide concepts and simulation results for the POWTEX instrument. Nuclear Instruments and Methods in Physics Research Section A: Accelerators, Spectrometers, Detectors and Associated Equipment, 680:124-133, July 2012.

[20] K. H. Klenø and K Lefmann. Optimization of supermirror guide coating. In preparation.

[21] Andreas K. Freund. Cross-sections of materials used as neutron monochromators and filters. Nuclear Instruments and Methods in Physics Research, 213(2-3):495-501, 1983.

[22] Peter K Willendrup, Linda Udby, Erik Knudsen, Emmanuel Farhi, and Kim Lefmann. Using McStas for modelling complex optics, using simple building bricks. Nuclear Instruments and Methods in Physics Research Section A: Accelerators, Spectrometers, Detectors and Associated Equipment, 634(1, Supplement):S150 - S155, 2011.

[23] Steve Peggs, editor. Conceptual Design Report. 2012.

[24] Kaspar Hewitt Klenø. Effects of Misalignment on Long Elliptical Guides. Report (unpublished), 2012.

[25] L.D. Cussen, D. Nekrassov, C. Zendler, Th. Krist, and K. Lieutenant. An Improved Elliptic Neutron Guide Design for ESS. In preparation.

[26] C. Zendler, K. Lieutenant, D. Nekrassov, L.D. Cussen, and M. Strobl. Bi-spectral beam extraction in combination with a focusing feeder. $\mathrm{Nu}$ - 
409

clear Instruments and Methods in Physics Research Section A: Accelerators, Spectrometers, Detectors and Associated Equipment, pages 1-8, December 2012. 